The University of San Francisco

USF Scholarship: a digital repository @ Gleeson Library | Geschke Center

9-28-2013

Antiretroviral Therapy (ART) Side Effect Impacted on Quality of Life, and Depressive Symptomatology: A Mixed-Method Study

Wei-Ti Chen

Cheng-Shi Shiu

Joyce Yang

Jane M. Simoni

Karen Fredriksen-Goldsen

See next page for additional authors

Follow this and additional works at: https://repository.usfca.edu/psyc

Part of the Psychology Commons 
Authors

Wei-Ti Chen, Cheng-Shi Shiu, Joyce Yang, Jane M. Simoni, Karen Fredriksen-Goldsen, Tony Szu-Hsien Lee, and Hongxin Zhao 


\title{
Antiretroviral Therapy (ART) Side Effect Impacted on Quality of Life, and Depressive Symptomatology: A Mixed-Method Study
}

\author{
Wei-Ti Chen ${ }^{1,}$, , Cheng-Shi Shiu ${ }^{2}$, Joyce P Yang ${ }^{3}$, Jane M Simoni ${ }^{3}$, karen I Fredriksen- \\ Goldsen ${ }^{4}$, Tony Szu-Hsien Lee ${ }^{5}$, and Hongxin Zhao ${ }^{6, *}$ \\ ${ }^{1}$ School of Nursing, Yale University, New Haven, CT \\ ${ }^{2}$ School of Social Service Administration, University of Chicago, Chicago, IL \\ ${ }^{3}$ Department of Psychology, University of Washington, Seattle, WA \\ ${ }^{4}$ School of Social Work, University of Washington, Seattle, WA \\ ${ }^{5}$ Department of Health Promotion and Education, National Taiwan Normal University, Taiwan \\ ${ }^{6}$ AIDS Unit, Beijing Ditan Hospital, Capital Medical University, Beijing, China
}

\begin{abstract}
Antiretroviral therapy (ART) is known for its side effects. In this paper, we describe ART side effects as experienced by Chinese HIV+ individuals. This study presents two stages of a research project, combining qualitative in-depth interviews (29 HIV+ participants) with quantitative statistical data analysis $(\mathrm{N}=120)$. All data was collected between July 2005 to March 2008 at Beijing's Ditan Hospital. Consent was obtained from each participant for the qualitative interview and again for the quantitative survey. During in-depth interviews, Chinese HIV+ patients reported experiencing digestive discomfort, skin rashes, numbness, memory loss, nightmares, and dizziness, which not only brought them physical discomfort, but also interrupted different dimensions of their social lives. Furthermore, multiple regression analyses revealed that those who reported more severe side effects also experienced greater depressive mood after controlling for other clinical and psychosocial factors. ART side effects are one of the primary reasons causing HIV+ individuals to delay or stop taking life-saving medication; therefore, clinical interventions are critically needed to assist HIV+ individuals in managing ART side effects. ART side effects reinforced existing negative attitudes toward ART and lead to lower ART adherence. Future research should focus on developing culturally sensitive interventions to enhance HIV+ selfmanagement, to alleviate physical and psychological burden from ART and HIV.
\end{abstract}

\section{Keywords}

Side effect; Antiretroviral therapy; China; HIV/AIDS; Depression; Quality of life

Copyright: (C) 2013 Chen WT, et al.

*Corresponding author: Wei-Ti Chen, Yale University, New Haven, CT, USA, Tel: 203-737-2416; wei-ti.chen@yale.edu, Zhao Hongxin, AIDS Unit, Beijing Ditan Hospital, Capital Medical University, Beijing, China 13911022130@163.com.

Citation: Chen WT, Shiu CS, Yang JP, Simoni JM, Fredriksen-Goldsen KI, et al. (2013) Antiretroviral Therapy (ART) Side Effect Impacted on Quality of Life, and Depressive Symptomatology: A Mixed-Method Study. J AIDS Clin Res 4: 218. doi: 10.4172/2155-6113.1000218 


\section{Background}

Today, in the United States, 30 antiretroviral therapies (ART) made up of 23 separate drugs are being used to control the HIV virus [1]. The growth in the number and kinds of medications available to treat HIV/AIDS has changed the prospects for HIV/AIDS management for both patients and providers. Treatment has gone from being a means of temporary delay in the face of imminent death, to sustained management of HIV/AIDS as a chronic, survivable disease [2]. Arrival of new treatment regimens, however, has come with new complications. Given the increasing potential for negative drug-to-drug interactions and the potential for multiple comorbidities, management of HIV patients has become a complex affair.

The Chinese national ART program, known as "Four Frees and One Care," began in 2003, and ART treatment is now widely available in China [3]. Initially, the only available antiretroviral drugs in China were domestically produced generic zidovudine (ZDV), stavudine (d4T), didanosine (ddI), and nevirapine (NVP). Approximately $80 \%$ of patients received ZDV + ddI + NVP. The other $20 \%$ received d4T + ddI + NVP, primarily due to hematologic contraindications or complications with ZDV. Domestically produced generic indinavir (IDV) and imported, branded efavirenz (EFV), and lamivudine (3TC) were added subsequently [4]. At present in China, although there are new medications available, overall regimens used in these combinations have not changed significantly since 2007 [5].

Before 2003, most Chinese HIV/AIDS patients on ART were using imported drugs they had obtained on their own. For example, friends might have brought the drugs back from overseas or purchased them on the black market. With early results of treatments provided by the "Four Frees and One Care" program [6], treatment was rapidly updated. By August 2008, more than 52,000 people had received first-line ART - about $97 \%$ of patients on medications in China were receiving free treatment through Four Frees and One Care [7]. At that time, the first-line treatment regimen of nucleoside reverse transcriptase inhibitors (NRTI) consisted of zidovudine (AZT) or stavudine (d4T) with nevirapine (NVP), all generically produced in China. The availability of free ART offered some flexibility in treating patients; however, an effective second-line regimen did not exist until 2008 [4]. Didanosine (ddI, a generic drug) was used as the third-line treatment until 2009, when the branded lamivudine (3TC) also became available [7]. Pare usually put on the first combination of AZT/D4T+3TC+NVP/EFV. Then, the provider will adjust the combination based on the patients' tolerance and side effects. The regimen might be changed to second line of the treatment if necessary [8].

Despite these successes in increasing treatment accessibility and outcomes, treatments for HIV have been well known for their severe side effects. For instance, the possible side effects of nevirapine (NVP) include severe gastrointestinal reactions, peripheral neuritis, and abnormal hepatic functions. Whitish plaques on the lips and bilateral buccal mucosa, as well as a burning sensation, taste disturbance, and xerostomia have also been reported [9]. Another first-line treatment, stavudine (d4T), is known for causing a variety of systemic discomforts, including mitochondrial toxicities (mainly peripheral neuropathies, lifethreatening lactic acidosis, and lipoatrophy) and metabolic dysfunctions (e.g. abdominal obesity, hypertriglyceridemia, low HDL cholesterol, high blood pressure, and high fasting glucose) [10]. These side effects are relatively frequent and worrisome, and have been reported often in studies [11]. However, satisfactory virological efficacy has been achieved in stavudine (d4T)-based combinations-d4T is usually combined with 3TC or NVP-and these combinations have diminished toxicities and side effects [11]. Unfortunately, some of these combinations might not be available in all parts of China at this time; therefore, 
Chinese patients taking ART may still need to tolerate side-effect syndromes of varying degrees, ranging from mild to life threatening.

Most of the studies related to side effects were conducted in Western countries, leaving a gap in the experiences of side effects among people living with HIV/AIDS (PLWHA) in countries with fewer resources, where generic drugs are integral parts of treatments. In one prospective study, all the Chinese HIV+ patients receiving generic drugs developed at least one episode of symptoms that could be attributed to treatment side effects, where about one third of which were considered clinically severe [12].

Although some HIV+ patients can overcome initial side effects, without appropriate treatments side effects may lead to challenging situations, including increasing stress and depression, decreasing adherence, and quality of life. ART adherence is affected by numerous psychological, social, and cultural factors. Indeed, several studies have reported that ART side effects are one of the major reasons that people living with HIV/AIDS (PLWHA) stop taking their HIV medication [13,14]. Interventions aiming to facilitate patients' self-management of side effects of ART could then help to maintain or improve adherence levels. The physical and psychological demands of coping with ART side effects can be overwhelming to patients and decrease their quality of life [15].

Based on empirical evidence, researchers concluded that despite the fact that ART treatments can reduce symptoms caused by HIV and other opportunistic infections, their effects on improving patients' quality of life may be "canceled" out by emerging toxicities and side effects experienced by patients [16]. Therefore, symptom management intervention for psychological distress and physiological fatigue should be key areas of focus to improve quality of life in HIV+ populations [17].

\section{Objectives}

Given high prevalence of the various side effects among Chinese HIV+ patients [18,19], in order to optimize AIDS care and probability of treatment success, it is necessary to understand how they experience ART side effects in their day-to-day life. In this study we aimed to explore how Chinese HIV+ patients experience ART side effects. Additionally, we tested the relationships between the burden of side effects, quality of life, and depressive symptomatology.

\section{Methods}

This study presents two stages of a research project. Qualitative in-depth interviews were conducted in the first stage (2005-2006) to explore different dimensions of treatment experiences among Chinese HIV+ patients; quantitative surveys were conducted in the second stage of the study (2006-2008) to test potential relationships informed by the results of the qualitative interviews. Both stages were conducted at Beijing's Ditan Hospital. Ditan Hospital is one of the premier treatment centers for infectious diseases in China. Participants who were receiving care from Ditan Hospital at their outpatient clinics as well as those who were recently discharged from the hospital were all invited to participate in the study. Since the overall ART regimens used in China have not changed much since 2007 [5], this study remains highly relevant to the current situation of the Chinese HIV+ population.

\section{Participants}

The Institutional Review Boards (IRB) at Ditan Hospital and the University of Washington approved all study procedures. Potential study participants were approached directly by clinic staff and informed about the study; those who were interested were then referred to 
research staff. To ensure fidelity across clinic staff, a project manager was onsite to rescreen the potential study participants after they were referred from clinical staff. After study personnel explained the nature, and potential risks and benefits of the study, potential participants who agreed to be part of the study gave written informed consent. Consent was obtained from each participant for the qualitative interview and again before the questionnaire was administered. Mandarin-speaking HIV+ patients were recruited separately for the qualitative ( $N=29)$ and quantitative $(N=120)$ stages of the study. Of the quantitative survey participants, 70 of them had been using ART for less than 3 months and 50 of them were taking ART longer than 3 months.

The inclusion criteria of the study were HIV+ patients on antiretroviral medications who are 18 years of age or older, Mandarin-speaking, and are receiving HIV-related care at Ditan Hospital in out-patient clinics or right before the participants were discharged from the AIDS floor. The exclusion criteria were physical incapacitation or psychological or cognitive impairment that would prevent their participation in the study as informally assessed by medical providers. These two stages were independently recruited from the same research site; therefore, participants may or may not have participated in both stages.

\section{Qualitative study}

For the qualitative interviews, participants could choose whether or not to have their interviews audio-recorded; if they declined, detailed notes were taken. All participants received $100 \mathrm{RMB}(\sim 16)$ for their participation in the semi-structured, in-depth interview (Table 1).

\section{Interview process}

We recruited 29 study participants in order to achieve what the authors determined as conceptual data saturation. Interviews took approximately 1 hour and were conducted in a private space at the hospital. Interviewers included Ditan Hospital research staff, physicians and nurses at the hospital, and researchers from the University of Washington. All interviewers were of Chinese background and conducted the interviews in Mandarin. Interviewers first completed a 2-day training course led by qualitative research experts at a local university to familiarize themselves with the goals of the study and to learn standardized procedures for qualitative interviewing. Interviewers used an interview guide to ensure that all of the following topics were discussed: HIV testing history, HIV serostatus disclosure experience, social support, medication history, side effects, and ART adherence (including medication access, facilitators and barriers to adherence, and self-efficacy). All interviews except one were audio-recorded, and all were transcribed into Mandarin verbatim.

\section{Data analysis}

Qualitative content analysis [20] and a commercial software package (Atlas.ti Version 6) were used to facilitate data coding and analysis. Two Chinese-speaking investigators who were trained in qualitative research methods independently reviewed the transcripts and identified codes to represent various themes in the narratives. These two investigators then reviewed and discussed their coding choices in order to resolve any discrepancies the meaning and assignment of codes, and general patterns observed in the data. Then, after codes were assigned and quotes were retrieved, the researchers presented general themes to the healthcare providers and community members at Ditan Hospital, who confirmed that these patterns were congruent with their experiences. This paper is based on a descriptive thematic analysis of the codes related to patient experiences with ART side effects. The goal is to explicate the range and meaning of related concepts in the participants' narratives by 
using descriptive thematic analysis. The selected quotations were translated into English by the two bilingual researchers who led this analysis.

\section{Quantitative study}

In the quantitative study, we aim to evaluate the relationship between the burden of side effects and depressive symptomatology. It is because if the Chinese PLWHA experienced in a very negative way in which side effects of HAART not only induced physical discomforts, but also influenced other domains of social life, it is very likely that the experiences of side effects may also associate with their depressive symptomatology. Therefore, we hypothesize that the greater burden the side effects experienced by the Chinese PLWHA, the more depressive symptomatology they would express.

For the quantitative portion, study participants were recruited from the outpatient clinic and AIDS patient floors connected to Ditan Hospital. Participants were required to be receiving care at the hospital and using ART at the time of the study. A total of 120 patients provided data. The study participants were recruited in the second stage which spanned across two years after completion of the first stage. The questionnaire was designed by the research team and was implemented in Chinese.

\section{Measures}

\section{Sociodemographic variables}

Participants' age, gender, marital/partner status, education level, income, residency, and employment status were collected via participant self-report.

\section{ART knowledge, quality of life, depressive symptomology, side effects, and social support}

Researchers assessed participants' knowledge about their medications using a validated HIV Knowledge scale [21], with three additional items adapted from a related project via an inperson interview [22]. Scores were averaged on a 0-3 scale, with Cronbach's $\alpha=0.73$. The quality of life was measured by the Medical Outcomes Study HIV Health Survey (MOSHIV) Chinese version. The MOS-HIV Chinese version was validated and had very good psychometrics properties (Cronhach's as ranged from 0.79 to 0.90) [23]. For the purpose of this study, we created two composite measures for physical and mental quality of life, following the standard procedures. The two composite measures both ranged from 0 to 100 . In addition, a revised 10-item version of the Center for Epidemiologic Studies Depression Scale (CES-D) was used, as described in a related paper [24]. Cronbach's a for the CES-D scale was 0.77 . Researchers also administered a 20 -item checklist via in-person interview to assess presence of side effects, which included a question evaluating whether side effects influence participants' daily lives, ranging from 0 (not at all) to 4 (very much). If participants indicated that they experienced side effects, we further explored the burden of the current side effects. "Current side effect severity" was determined by an item that ranged from 0 (not at all) to 4 (very much). Finally, researchers administered a 14-item checklist via in-person interview to assess the types of social support participants had received from their caregivers. The 14 items were added to derive an aggregate measure of the level of social support (0-14). Cronbach's a for the social support scale was 0.75 .

Missing values comprised about $10 \%$ of the sample. We have investigated the missing mechanisms and concluded that "missing at random (MAR)" is a reasonable assumption for this dataset, so we performed multiple imputations to handle the missing values. All the statistical analysis was performed in STATA version 12, commercial statistical software. 


\section{Results}

\section{Qualitative findings}

The sample comprised 29 HIV+ patients, of whom 7 were women and 22 were men. Most of the sample $(n=26,90 \%)$ were ethnically Han, an ethnic group native to East Asia, and a majority $(n=22,76 \%$ ) lived in Beijing. The mean age of the study participants was 38 years. Many had at least a high school education $(n=20,72 \%)$. Some of the participants $(n=11$, $38 \%$ ) did not reveal their HIV transmission routes. Among those who did, sexual contact $(n=10,34 \%)$ and blood-to-blood transmission $(n=8,28 \%)$ were the most common means of transmission. Participants' length of awareness of their HIV status ranged from 2 to 10 years. More than half $(n=11,59 \%)$ were married, and four reported that they were living with a steady same-sex partner. Additional details of the population have been published [25].

Twenty-one of these had been taking ART for longer than 6 months, and the other eight had started ART within the previous 2 weeks. Several ART side effects were reported by the study participants, including those with physiological and psychological symptoms. Below are some of the ART-related discomforts that were often mentioned.

Digestive system-Digestive system issues like diarrhea, dry mouth, nausea, vomiting, and weight loss/gain were most frequently mentioned. Although digestive side effects were mild for most participants, for a few, the frequency and intensity were so severe that they would use terms like "totally empty" to describe their state of mind after a serious episode. Side effects this severe may even interfere with a patient's food intake and treatment decisions. A 40-year-old male participant vividly shared his experience of diarrhea

I had severe diarrhea and lost a lot of weight. I must be dying. I couldn't keep anything down and I didn't want to eat anything. [I kept eating] just because my family kept telling me to think about my children; otherwise, I would not have wanted to stay in the hospital and deal with the ART side effects.

The size of ART pills can also exacerbate a patient's digestive side effects. For example, the didanosine (ddI) tablet is large to swallow, so to take this medication, one need to first chew the tablet, during which time the taste and odor may trigger a nauseous reaction. As attested by one 66-year-old patient "The pill is too big. After I chew that big tablet, I feel very nauseous and have a stomach ache."

Pancreatitis-A more serious kind of side effect impacted a few patients. It has been well documented that some HIV medications, such as didanosine (ddI) and stavudine (d4T), can cause more severe side effects like pancreatitis, which results from the interaction between the ingredients in the medicine and certain digestive enzymes [26,27]. Without appropriate treatment, pancreatitis can be lethal. A 30-year-old male described his experiences, which started with acute abdominal pain:

I was suffering severe pain from noon to midnight. I couldn't tolerate it anymore, so I came into the emergency room here. I was ... diagnosed with pancreatitis. They [physicians] checked my enzymes. Some of the enzymes were normal and some were not ... They all believed these symptoms were side effects from ART. And I heard that chewing the tablet in my [ART] combination is bad for the pancreas.

The pain caused by pancreatitis can be so intense that it can overwhelm the ability of narcotics to suppress it. One interviewee perceived his experiences of hospitalization through the prism of pain brought on by acute pancreatitis: "I was constantly given the pain 
medicine," he said, "and I still remember that the pain kept me awake, even with all those narcotics."

Dermatological symptoms-The second most complained about side effect was skin issues, including, but not limited to, change of skin tone, skin rashes, and hair loss. For instance, a 37-year-old female told us that after she started ART, she noticed her skin turning black and looking like charcoal. The darkening of skin tone occurred not just on her trunk but also on her face, which was understandably very distressing to her. Other skinrelated side effects included uncomfortable sensations and itches. Participants complained of a burning and tingling sensation all over their bodies. Besides the physical discomfort they cause, skin-related side effects may have significant implications for patients' social lives, especially in China, where HIV stigma is still rampant. Visible changes to a patient's skin may be perceived by others as a "mark of disease" and may solicit unwanted attention. One 42-year-old male participant decided to wear long sleeves constantly, even during summer, because of skin rashes on his arms. During his interview, which occurred in late summer, he pointed to his unseasonal clothing, said:

Did you wonder why I am not wearing short sleeves? It is because those scars are still there, so I can only wear long-sleeved shirts. I wish summer would go faster, especially when I see my coworkers wearing shorts and tank tops, while I have to have my long sleeves on. People keep asking me: "Hey, don't you feel hot?" I always tell them that I am allergic to sunlight.

Neurological symptoms-Many study participants also complained of numbness, using a variety of illuminating metaphors to describe their feelings. Among these was "a sensation of electric shock," "being frozen," "like wearing tight shoes," and "walking barefoot on a gravel path." While many of the participants stated that the numbness was usually constant and long lasting, it could become worse during sleep. The feeling of numbness could be mingled with other unpleasant sensations, such as soreness and throbbing pain, which prevented patients from getting restful sleep at night. One 46-year-old male participant described how the spreading and encroaching numbness had caused him insomnia for several nights:

The numbness crept up from my feet and spread out over my body. Just like with an electrical shock, the feet feel it the worst. During the night, my feet are so sore, numb, and swollen that I can hardly sleep. The pain is so constant. I can hardly close my eyes.

Numbness can interrupt patients' daily activities in serious ways. Because it affects mostly patients' feet and hands, it may reduce their ability to carrying out their job duties. This is exemplified by the description of a 32-year-old female participant, who stated that she was "suffering from side effects [numb feet] every minute of every day." Because the side effects were very serious in her case, she was confined to her bed and could not work to support herself. To alleviate numbness, interviewees tried different strategies, including massage therapy and wearing softer shoes, although most of these efforts were in vain. Some patients had to change their regimens to get relief from the side effects.

Memory loss, nightmares, and dizziness-Some of the patients complained about symptoms associated with the central nervous system (CNS), including memory loss, nightmare, and dizziness. A 28-year-old male participant stated that he used to have a sharp memory, but after he initiated ART treatment, he began suffering from partial memory loss. A 37-year-old female patient said that when she took efavirenz (EFV), she was so dizzy she could hardly stand. To her it was as if "the sky was falling down." She also began to have nightmares that significantly decreased her sleep quality. As a result, she had to spend most 
of her time in bed and so was unable to work. Another 66-year-old male participant echoed her experience. This participant had suffered from dizziness as an ART side effect for more than 6 months. The dizziness was so bad it interfered with his work, he told the interviewer. "I feel so light. My brain is empty, and I have no energy to do anything. This is not the way it should be."

\section{Quantitative findings}

A total of 142 eligible participants were invited to participate in the study, and 120 of them agreed to participate, for an $85 \%$ recruitment rate. The sample was $82 \%(N=98)$ male, with a mean age of 36 years $(S D=8.0)$. Slightly more than half $(54 \% ; N=65)$ of the participants were working, and 55\% ( $N=66)$ reported that they were married or had a steady partner. Nearly all study participants $(93 \% ; N=112)$ were of Han ethnicity, and close to two thirds $(62 \% ; N=74))$ had finished high school. Detailed demographic information is presented in Table 2.

Within this sample, 53.3\% ( $N=64)$ had experienced digestive side effects; $51.7 \%(N=62)$ had skin-related symptoms; $36.1 \%(N=43)$ had numbness in hands and feet; and $73.6 \%$ $(N=88)$ had memory loss, nightmares, and/or dizziness. Because pancreatitis requires official diagnosis from a physician, we did not track this information in the dataset; however, $41.9 \%(N=50)$ of our sample reported experiencing some kind of stomach ache in connection with the ART.

We conducted bivariate analysis to explore the relationships between depressive mood, burden of side-effects, psychosocial factors, demographic data, CD4 values, and medication combinations. Detailed information related to side-effect severity is presented in Table 2. Then, we used multivariate multiple linear regression to further investigate the relationship between depressive mood, quality of life, and perceived burden of side effects, adjusting for the effects of covariates and confounders, such as stigma, ART knowledge, and social support. As expected, the distribution of depression (CES-D) scores was highly skewed, so the Yeo-Johnson procedure was used to transform the dependent variable to make its distribution more reasonably normal. The scores for the physical and mental quality of life had reasonably normal distributions. The results of the model fitting are presented in the Table 3. Except for the finding that age is marginally significantly related to CES-D, none of the demographic and clinical factors were related to the CES-D scores. Although ART knowledge significantly related to CES-D scores in the bivariate analysis, the relationship was no longer significant when other factors were controlled for. By contrast, the perceived burden of side effects was significantly associated with CES-D scores. Finally, social support and general health also had significant relationships with a patient's depressive mood, although they were entered into the model only to adjust the relationship between CES-D and side effects and were not our primary interest.

\section{Discussion}

In the current study, we documented how Chinese PLWHA experienced side effects of ART. These side effects not only brought physical pain and discomfort but also affected many aspects of daily life. Certain CNS-related discomforts induced by ART-conditions such as memory loss, dizziness, and numb feet- could, in their more extreme forms, impinge upon Chinese PLWHA's daily functioning and prevent them from conducting regular activities necessary to support themselves and their families. These symptoms are similar to those reported by other studies conducted in China [18].

In addition, many Chinese PLWHAs in this study expressed that therapy-related alterations in their appearance can exacerbate existing HIV stigma to increase psychological burden. As 
a result, they may need to fabricate reasonable excuses to explain their changing appearance or the unseasonable clothing they may use to hide their dermatological symptoms. Given that ART side effects could interfere with a wide range of dimensions of daily life among Chinese PLWHA, depression can be another pernicious consequence of ART side effects.

From this study, surprisingly, none of the demographic variables, CD4 values, or medication combinations had significant relationships with depressive mood. In contrast, all of the psychosocial factors, except for HIV stigma, had significant relationships with depressive mood. In particular, patients who received more social support, had greater knowledge of their medications, and enjoyed better general health, were less depressed. In addition, those who reported greater burden of side effects tended to report more depressed mood.

As ART side effects have the capacity to bring Chinese PLWHA many challenges that have significant social and cultural implications, it is expected that those facing greater burdens of side effects would experience greater depressive mood. Although the relationship between depressive symptomatology and experienced ART side effects has not been a traditional research focus, our findings are consistent with existing literature. Low-Beer and colleagues found that participants' depressive symptomatology deteriorated after they initiated protease inhibitors-based treatments [28]. This was especially salient for those who enjoyed higher quality of life before their treatments; their depressive symptomatology deteriorated along with three other dimensions of quality of life (physical, social, and role functions) [28]. Another study also reported that personal experience of ART-related side effects and depression were highly associated in HIV+ individuals [29]. Thus, developing clinical interventions that address self-management of side effects is greatly needed. Interventions that focus on psychosocial factors, enhance protective health routines, and reduce risk behaviors (e.g. drug holidays) are likely to lessen overall morbidity and mortality among patients living with HIV/AIDS [30]. Therefore, providing a manual of self-management for ART side effects could help patients deal with the discomfort of these side effects. At the same time, it could decrease side-effect related depression.

In Chinese culture, the concept of health encompasses fulfillment of obligations to support family. Not being able to carry out one's duties or to maintain harmonious relationships with others in one's social network is considered to be socially "unhealthy" in Chinese culture [31]. Consequently, there is a significant social cost to ART side effects in addition to the physical and psychological ones. Compromised social functioning induced by ART side effects may further render some Chinese PLWHA to lose faith in their regimens while driving some others to seek help from Complementary and Alternative Medicine (CAM) [14,32]. Since allopathic medicine (like ART) is thought by many Chinese individuals to cause disruption in the body's yin/yang balance, it is believed to cause harm to the body, and this harm is seen as being evidenced by side-effects. In fact, for many Chinese PLWHA, ART is seen as being akin to poison [14]. Experience of side effects can thus significantly predict Chinese PLWHA's preference for CAM over ART. Many Chinese PLWHA who embrace allopathic medicine and ART may also use CAM to manage their experienced sideeffects [14]. They believe that CAM can compensate for the toxicity and yin/yang imbalance caused by ART and can therefore decrease side effects [14].

Currently, metabolic syndromes (e.g. abdominal obesity, hypertriglyceridemia, low HDL cholesterol, high blood pressure, and high fasting glucose) related to ART use are rarely addressed in developing countries like China. These side effects have caused PLWHAs in China to change behaviors including using CAM more frequently and altered their self image. Switching drugs (for example, switching stavudine (d4T) to another Nucleoside Reverse Transcriptase Inhibitor (NRTI)) would be one acceptable alternative that could be used to alleviate such syndromes. However, clinicians need to confirm the availability of 
alternative NRTIs in their particular facility, country, or region before proposing a change between medicines or combinations. Behavior and diet changes can moderate side effects as well, which are especially attractive alternatives in resource-limited settings, where drugswitching options are limited [11]. For example, efforts as simple as getting regular exercise and limiting high-cholesterol foods can prevent dyslipidemic side effects [33]. Studies have shown that symptom management interventions have been effective at increasing self-care ability in managing medication side effects with HIV+ individuals [34]. Also, depression and catastrophizing pain were proofed as important factors in managing HIV-related neuropathy and ART adherence [35]. Furthermore, Mediterranean-style diets (e.g., eating primarily plant-based foods, such as fruits and vegetables, whole grains, legumes and nuts, replacing butter with healthy fats, such as olive oil, using herbs and spices instead of salt to flavor foods, limiting red meat to no more than a few times a month, eating fish and poultry at least twice a week or drinking red wine in moderation (optional)) were found to be effective in improving the HIV-associated lipodystrophy syndrome [36]. Thus, we recommend that self-management programs for side effects be applied in clinical practice generally.

\section{Limitations}

There were several limitations in this study. First, we recruited only at one large hospital, in Beijing, China. Since Beijing is a metropolitan area and the capital city, it has better resources for Chinese PLWHA than most other areas. Second, as the disease progress from HIV to AIDS, patients might develop opportunistic diseases whose symptoms (for example, fatigue and sleeplessness) can mimic ART side effects. As researchers, we were not always able to differentiate between symptoms related to disease progression and ART side effects. Third, by the time of the quantitative phase of the study, some of the participants had been using ART for longer than 6 months, and they might not have remembered some of the initial ART side effects that they experienced. Some of them who had skipped this part of the questionnaire may have done so in error, thinking that they had not experienced side effects, when in fact they had. Fourth, the acceptable internal consistency score on several scales used in this project indicate the cultural adaptations of these scales need to be further investigated. Last, the Chinese government was aware of these side effects from ART, thus, efforts were made to change the formulation of first-line ART treatment in order to decrease side effects.

\section{Conclusion}

As we discovered in this study, the symptoms of ART side effects have biological, psychological, and social dimensions that impact Chinese PLWHA in unique ways. To some extent, ART side effects can be moderated, but even so, side effects remain a part of the comprehensive illness experience. Without careful management, side effects can reinforce existing negative attitudes and ultimately lead to lower ART adherence levels. Future research should focus on developing culturally sensitive interventions to enhanced selfmanagement that can alleviate the physical and psychological burdens from ART and from the disease.

\section{Acknowledgments}

This study was supported in part by MH074364 and MH074364-S1 from the National Institute of Mental Health (Simoni, PI). We acknowledge Bu Huang for her assistance in obtaining funding for the project, Xin Wang and Wei $\mathrm{Qu}$ for coordinating the study onsite. In addition, this publication was supported (in part) from research supported by an NIH funded program (5R25MH087217-03 Barbara Guthrie \& Jean Schensul (PIs) NIH-NIMH, Research Education Institute for Diverse Scholars (REIDS) Center for Interdisciplinary Research on AIDS (CIRA) and 1K23NR014107; PI: Wei-Ti Chen). 


\section{References}

1. Alozie OK, Bonham S, Henry WK. Management of HIV: a swing back to the future. Minn Med. 2009; 92:45-49. [PubMed: 19916274]

2. Inouye J, Boland MG, Nigg CR, Sullivan K, Leake A, et al. A center for self-management of chronic illnesses in diverse groups. Hawaii Med J. 2011; 70:4-8. [PubMed: 21225588]

3. Zhang FJ, Pan J, Yu L, Wen Y, Zhao Y. Current progress of China's free ART program. Cell Res. 2005; 15:877-882. [PubMed: 16354563]

4. Zhang F, Haberer JE, Wang Y, Zhao Y, Ma Y, et al. The Chinese free antiretroviral treatment program: challenges and responses. AIDS. 2007; 21(Suppl 8):S143-148. [PubMed: 18172383]

5. Zhang F, Dou Z, Ma Y, Zhang Y, Zhao Y, et al. Effect of earlier initiation of antiretroviral treatment and increased treatment coverage on HIV-related mortality in China: a national observational cohort study. Lancet Infect Dis. 2011; 11:516-524. [PubMed: 21600849]

6. Cao YZ, Lu HZ. Care of HIV-infected patients in China. Cell Res. 2005; 15:883-890. [PubMed: 16354564]

7. Zhang F, Dou Z, Ma Y, Zhao Y, Liu Z, et al. Five-year outcomes of the China National Free Antiretroviral Treatment Program. Ann Intern Med. 2009; 151:241-251. W-52. [PubMed: 19687491]

8. Zhang, F. Naitonal free antiretroviral therapy treatment guideline [Chinese]. Beijing Huilin publishing company; Beijing: 2012.

9. Moura MD, Senna MI, Madureira DF, Fonseca LM, Mesquita RA. Oral adverse effects due to the use of Nevirapine. J Contemp Dent Pract. 2008; 9:84-90. [PubMed: 18176653]

10. Jericó C, Knobel H, Montero M, Ordoñez-Llanos J, Guelar A, et al. Metabolic syndrome among HIV-infected patients: prevalence, characteristics, and related factors. Diabetes Care. 2005; 28:132-137. [PubMed: 15616246]

11. Makinson A, Moing VL, Kouanfack C, Laurent C, Delaporte E. Safety of stavudine in the treatment of HIV infection with a special focus on resource-limited settings. Expert Opin Drug Saf. 2008; 7:283-293. [PubMed: 18462186]

12. Li T, Dai Y, Kuang J, Jiang J, Han Y, et al. Three generic nevirapine-based antiretroviral treatments in Chinese HIV/AIDS patients: multicentric observation cohort. PLoS One. 2008; 3:e3918. [PubMed: 19081791]

13. Simoni JM, Pantalone DW, Plummer MD, Huang B. A randomized controlled trial of a peer support intervention targeting antiretroviral medication adherence and depressive symptomatology in HIV-positive men and women. Health Psychol. 2007; 26:488-495. [PubMed: 17605569]

14. Chen WT, Shiu CS, Simoni J, Fredriksen-Goldsen K, Zhang F, et al. Attitudes toward antiretroviral therapy and complementary and alternative medicine in Chinese patients infected with HIV. J Assoc Nurses AIDS Care. 2009; 20:203-217. [PubMed: 19427598]

15. Johnson MO, Stallworth T, Neilands TB. The drugs or the disease? Causal attributions of symptoms held by HIV-positive adults on HAART. AIDS Behav. 2003; 7:109-117. [PubMed: 14586196]

16. Burgoyne RW, Tan DH. Prolongation and quality of life for HIV-infected adults treated with highly active antiretroviral therapy (HAART): a balancing act. J Antimicrob Chemother. 2008; 61:469-473. [PubMed: 18174196]

17. Yeh ML, Liu HY, Ko WC, Lee HC, Ko NY. [HIV-related symptoms in patients with HIV infection enrolled in an HIV case management program in Taiwan]. Hu Li Za Zhi. 2009; 56:3542. [PubMed: 19221999]

18. He M, Zheng YH, Zhou HY, Mamadou D, Chen Z, et al. Prospective observation for seven-year's highly active antiretroviral therapy in Chinese HIV-1 infected patients. Curr HIV Res. 2011; 9:160-165. [PubMed: 21585332]

19. Zhou H, Zheng Y, He Y, Gong G, Chen Z, et al. Therapeutic effect and safety evaluation on 6-year highly active antiretroviral therapy for Chinese HIV-1 infected patients. Zhong Nan Da Xue Xue Bao Yi Xue Ban. 2009; 34:731-737. [PubMed: 19734579]

20. Hsieh HF, Shannon SE. Three approaches to qualitative content analysis. Qual Health Res. 2005; 15:1277-1288. [PubMed: 16204405] 
21. Remien RH, Smith RA. HIV prevention in the era of HAART: implications for providers. AIDS Read. 2000; 10:247-251. [PubMed: 10808608]

22. Simoni JM, Chen WT, Huh D, Fredriksen-Goldsen KI, Pearson C, et al. A preliminary randomized controlled trial of a nurse-delivered medication adherence intervention among HIV-positive outpatients initiating antiretroviral therapy in Beijing, China. AIDS Behav. 2011; 15:919-929. [PubMed: 20957423]

23. Lau JT, Tsui HY, Patrick LC, Rita CW, Molassiotis A. Validation of a Chinese version of the Medical Outcomes Study HIV Health Survey (MOSHIV) among Chinese people living with HIV/ AIDS in Hong Kong. Qual Life Res. 2006; 15:1079-1089. [PubMed: 16900288]

24. Rao D, Chen WT, Pearson CR, Simoni JM, Fredriksen-Goldsen K, et al. Social support mediates the relationship between HIV stigma and depression/quality of life among people living with HIV in Beijing, China. Int J STD AIDS. 2012; 23:481-484. [PubMed: 22844001]

25. Starks H, Simoni J, Zhao H, Huang B, Fredriksen-Goldsen K, et al. Conceptualizing antiretroviral adherence in Beijing, China. AIDS Care. 2008; 20:607-614. [PubMed: 18576162]

26. Lai HY, Chen JH, Tsai PP, Ho MW, Shen WC. Hepatic steatosis and pancreatitis associated with the use of stavudine in a patient with HIV infection. AJR Am J Roentgenol. 2004; 183:1605-1607. [PubMed: 15547198]

27. Gerschenson M, Brinkman K. Mitochondrial dysfunction in AIDS and its treatment. Mitochondrion. 2004; 4:763-777. [PubMed: 16120431]

28. Low-Beer S, Chan K, Wood E, Yip B, Montaner JS, et al. Health related quality of life among persons with HIV after the use of protease inhibitors. Qual Life Res. 2000; 9:941-949. [PubMed: 11284213]

29. Herrmann S, McKinnon E, John M, Hyland N, Martinez OP, et al. Evidence-based, multifactorial approach to addressing non-adherence to antiretroviral therapy and improving standards of care. Intern Med J. 2008; 38:8-15. [PubMed: 17916167]

30. Gore-Felton C, Koopman C. Behavioral mediation of the relationship between psychosocial factors and HIV disease progression. Psychosom Med. 2008; 70:569-574. [PubMed: 18519885]

31. Lew-Ting CY, Chen LH. The surrogate marker and its discontents: pluralism in immunity maintenance among HIV-infected persons in Taiwan. Sociol Health Illn. 2008; 30:1039-1054. [PubMed: 18764805]

32. Chen, WT. Complementary and alternative medicine and traditional Chinese medicine in a Chinese population who are HIV-positive: Perceptions and beliefs, in HIV in Communities of Color: The Compendium of Culturally Competent Promising Practices: The Role of Traditional Healing in HIV Clinical Management.. In: Downer, G., editor. National Minority AIDS Education and Training Center; Washington, DC: 2011. p. 13-20.

33. Montoya CJ, Jaimes F, Higuita EA, Convers-Páez S, Estrada S, et al. Antiretroviral effect of lovastatin on HIV-1-infected individuals without highly active antiretroviral therapy (The LIVE study): a phase-II randomized clinical trial. Trials. 2009; 10:41. [PubMed: 19538732]

34. Chiou PY, Kuo BI, Lee MB, Chen YM, Chuang P, et al. A programme of symptom management for improving quality of life and drug adherence in AIDS/HIV patients. J Adv Nurs. 2006; 55:169-179. [PubMed: 16866809]

35. Lucey BP, Clifford DB, Creighton J, Edwards RR, McArthur JC, et al. Relationship of depression and catastrophizing to pain, disability, and medication adherence in patients with HIV-associated sensory neuropathy. AIDS Care. 2011; 23:921-928. [PubMed: 21500021]

36. Loonam CR, Mullen A. Nutrition and the HIV-associated lipodystrophy syndrome. Nutr Res Rev. 2012; 25:267-287. [PubMed: 23174511] 
Table 1

Qualitative In-depth Interview Schedule.

\begin{tabular}{|c|c|}
\hline Warm up & Let's start by having you tell me a little about yourself. How are you feeling today? How has your health been lately? \\
\hline & $\begin{array}{l}\text { Could you tell me something about your experience with HIV/AIDS? } \\
\text { Could you tell me something about your experience with HIV medications? } \\
\text { From your understanding, how do Anti-retroviral medications work? } \\
\text { What do you think will make it hard for you to take your medications? Or what has made it hard in the past? } \\
\text { Some circumstances that may make it difficult for some people to take medications. Let me ask how you think these would apply } \\
\text { to you. } \\
\text { a. Side effects from HIV medications } \\
\text { b. Depression: Can you describe a time when you (your partner) felt sad, felt like you (he/she) did not want to eat, or experienced } \\
\text { feelings of depression? If so please tell me about you (your partner) experience. } \\
\text { a. Stigma (having to hide) } \\
\text { b. Access to medication or medical care } \\
\text { c. Financial problems } \\
\text { Finally, please tell us a little about yourself? For example.... } \\
\text { a. Where are you from, what province? } \\
\text { b. What is your ethnicity? } \\
\text { c. What is your age? } \\
\text { d. What was the last year you finished ofschool? } \\
\text { e. What was your last job? } \\
\text { f. Who lives in your household? } \\
\text { g. At home do you have your own room or do you share a room with others? } \\
\text { h. Do you have a male or female spouse or partner? } \\
\text { i. Is there anything else you would like to share with us about using HIV medications? }\end{array}$ \\
\hline Closing & Ok. That's it for our questions. Do you have anything else to add or anything else to ask me? \\
\hline
\end{tabular}


Table 2

Sample Background Characteristics and their Relationship to Quality of Life and CESD, $(N=120)$.

\begin{tabular}{|c|c|c|c|c|c|c|c|}
\hline & \multirow{2}{*}{$\begin{array}{l}\text { Total } \\
\text { Percentage }(N)\end{array}$} & \multicolumn{2}{|c|}{ Physical QoL } & \multicolumn{2}{|c|}{ Mental QoL } & \multicolumn{2}{|c|}{ CES-D } \\
\hline & & Mean & SE & Mean & SE & Mean & SE \\
\hline \multicolumn{8}{|l|}{ Gender } \\
\hline Female & $18.3(22)$ & 43.94 & 2.91 & 45.67 & 2.32 & 3.61 & 0.42 \\
\hline Male & $81.7(98)$ & 45.94 & 1.11 & 49.03 & 1.08 & 3.54 & 0.18 \\
\hline \multicolumn{8}{|l|}{ Education } \\
\hline$<\mathrm{HS}$ & $38.3(46)$ & 48.35 & 1.59 & 48.68 & 1.47 & 3.35 & 0.28 \\
\hline HS & $37.5(45)$ & 44.58 & 1.71 & 49.16 & 1.71 & 3.65 & 0.28 \\
\hline$>\mathrm{HS}$ & $24.2(29)$ & $42.70^{*}$ & 2.11 & 46.81 & 2.15 & 3.75 & 0.31 \\
\hline \multicolumn{8}{|l|}{ Current Residency } \\
\hline Urban & $63.3(76)$ & 44.64 & 1.23 & 48.67 & 1.28 & 3.50 & 0.21 \\
\hline Suburban & $17.5(21)$ & 46.41 & 2.51 & 49.11 & 2.25 & 3.64 & 0.33 \\
\hline Rural & $19.2(23)$ & 47.88 & 2.44 & 46.93 & 2.16 & 3.67 & 0.40 \\
\hline \multicolumn{8}{|l|}{ Work Status } \\
\hline Professional & $33.9(41)$ & 44.94 & 1.95 & 48.05 & 1.66 & 3.50 & 0.26 \\
\hline Manual Labor & $15.0(18)$ & 48.86 & 3.06 & 47.47 & 2.36 & 3.18 & 0.46 \\
\hline Freelancer & $19.4(23)$ & 46.75 & 1.29 & 48.99 & 1.83 & 3.55 & 0.32 \\
\hline Not Working & $31.7(38)$ & 44.49 & 2.00 & 49.38 & 2.04 & 3.73 & 0.34 \\
\hline \multicolumn{8}{|l|}{ Relationship Status } \\
\hline Married/Partnered & $55.0(66)$ & 44.27 & 1.69 & 47.06 & 1.48 & 3.79 & 0.23 \\
\hline Other & $45.0(54)$ & 46.63 & 1.34 & 49.51 & 1.32 & 3.37 & 0.23 \\
\hline \multicolumn{8}{|l|}{ Household Income } \\
\hline < $2000 \mathrm{RMB} /$ month & $41.7(50)$ & 46.58 & 1.56 & 47.99 & 1.27 & 3.55 & 0.23 \\
\hline > $2001 \mathrm{RMB} /$ month & $58.3(70)$ & 44.57 & 1.32 & 49.37 & 1.56 & 3.52 & 0.24 \\
\hline \multicolumn{8}{|l|}{ Time on ART } \\
\hline$=<6$ months & $58.3(70)$ & 49.53 & 1.06 & 48.66 & 1.12 & 3.41 & 0.21 \\
\hline$>6$ months & $41.7(50)$ & $40.02^{* *}$ & 1.90 & 48.06 & 1.80 & 3.76 & 0.26 \\
\hline \multicolumn{8}{|l|}{ ART Combination } \\
\hline $\mathrm{AZT}+\mathrm{NVP}+3 \mathrm{TC}$ & $52.5(63)$ & 45.85 & 1.49 & 48.20 & 1.30 & 3.64 & 0.23 \\
\hline $\mathrm{AZT}+\mathrm{EFV}+3 \mathrm{TC}$ & $22.5(27)$ & 48.77 & 1.70 & 48.17 & 1.88 & 3.59 & 0.31 \\
\hline All others & $25.0(30)$ & 42.60 & 2.19 & 49.66 & 2.46 & 3.40 & 0.38 \\
\hline \multicolumn{8}{|l|}{ CD4 Rank } \\
\hline$<200$ & $40.0(48)$ & 44.65 & 1.51 & 48.93 & 1.53 & 3.68 & 0.22 \\
\hline $200-350$ & $36.7(44)$ & 46.73 & 1.99 & 48.36 & 1.82 & 3.39 & 0.31 \\
\hline $351-500$ & $16.7(20)$ & 47.53 & 2.05 & 50.96 & 2.31 & 3.23 & 0.52 \\
\hline \multirow[t]{2}{*}{$>500$} & $6.6(8)$ & 45.83 & 4.48 & 45.67 & 4.30 & 3.73 & 0.58 \\
\hline & Mean (SD) & $\beta$ & SE & $\beta$ & $\mathrm{SE}$ & $\beta$ & SE \\
\hline
\end{tabular}




\begin{tabular}{|c|c|c|c|c|c|c|c|}
\hline & \multirow{2}{*}{$\begin{array}{l}\text { Total } \\
\operatorname{Percentage}(N)\end{array}$} & \multicolumn{2}{|c|}{ Physical QoL } & \multicolumn{2}{|c|}{ Mental QoL } & \multicolumn{2}{|l|}{ CES-D } \\
\hline & & Mean & SE & Mean & SE & Mean & $\mathbf{S E}$ \\
\hline Age & $36.3(.73)$ & 0.03 & 0.13 & 0.17 & 0.12 & $-0.03^{\dagger}$ & 0.02 \\
\hline Social Support & $51.3(1.8)$ & $0.19^{* *}$ & 0.05 & $0.15^{* *}$ & 0.05 & $-0.02^{* *}$ & 0.01 \\
\hline HIV Stigma & $24.8(.57)$ & $-0.36^{*}$ & 0.16 & -0.17 & 0.17 & 0.03 & 0.03 \\
\hline ART Knowledge & $2.08(.03)$ & 3.33 & 3.04 & $10.07^{* *}$ & 2.88 & $-0.84^{\dagger}$ & 0.49 \\
\hline Side-Effect Severity & $2.00(.07)$ & $-7.46^{* *}$ & 1.23 & $-7.04^{* *}$ & 1.28 & $1.12^{* * *}$ & 0.20 \\
\hline
\end{tabular}

Physical QoL = Physical Quality of Life; Mental QoL = Mental Quality of Life; CES-D = Transformed Center for Epidemiologic Studies Depression Scale; $\mathbf{S E}=$ Standard Error; HS = High school; RMB = Renminbi, Chinese unit of currency;Zidovudine (AZT);Nevirapine (NVP); lamivudine (3TC); Efavirenz (EFV).

***

$\boldsymbol{P}<0.01$

* $<<0.05$

$t_{P}<0.1$ 
Table 3

Predictors of Quality of Life and CESD among 120 HIV-Positive Individuals in China.

\begin{tabular}{|l|c|c|c|c|c|c|}
\hline & \multicolumn{2}{|c|}{ Physical QoL } & \multicolumn{2}{c|}{ Mental QoL } & \multicolumn{2}{|c|}{ CES-D } \\
\hline & $\boldsymbol{\beta}$ & SE & $\boldsymbol{\beta}$ & SE & $\boldsymbol{\beta}$ & SE \\
\hline Constant & $63.38^{* *}$ & 8.71 & $41.18^{* *}$ & 10.78 & 2.81 & 1.78 \\
\hline Age & 0.06 & 0.12 & 0.19 & 0.14 & $-0.04^{\dagger}$ & 0.02 \\
\hline Gender & & & & & & \\
\hline Female vs. Male & 2.86 & 2.69 & $5.07^{\dagger}$ & 2.74 & -0.47 & 0.47 \\
\hline Time on ART & & & & & & \\
\hline =<6 months vs. > 6 months & $-7.88^{* *}$ & 2.28 & 0.19 & 2.29 & 0.27 & 0.39 \\
\hline ART Combination & & & & & & \\
\hline AZT + NVP + 3TC vs. AZT + EFV + 3TC & -1.68 & 2.26 & $-4.89^{\dagger}$ & 2.62 & $0.87^{\dagger}$ & 0.46 \\
\hline AZT + NVP + 3TC vs. All others & 0.55 & 2.12 & 0.89 & 2.52 & -0.35 & 0.43 \\
\hline CD4 Rank & & & & & & \\
\hline < 200 vs. 200 - 350 & -1.02 & 2.16 & -3.26 & 2.40 & 0.12 & 0.41 \\
\hline < 200 vs. 351 - 500 & -2.57 & 2.66 & -2.36 & 2.99 & 0.28 & 0.53 \\
\hline < 200 vs. > 500 & 0.07 & 3.41 & -4.54 & 3.86 & 0.01 & 0.68 \\
\hline ART Knowledge & -1.22 & 2.73 & 4.19 & 3.21 & 0.05 & 0.57 \\
\hline Social Support & $-0.13^{*}$ & 0.05 & $0.12^{*}$ & 0.06 & $-0.02^{*}$ & 0.01 \\
\hline HIV Stigma & $-6.85^{* *}$ & 1.23 & $-6.35^{* *}$ & 1.40 & $1.12 * *$ & 0.24 \\
\hline Side-Effect Severity & & -0.18 & 0.18 & 0.03 & 0.03 \\
\hline B E E & & & & & & \\
\hline
\end{tabular}

$\boldsymbol{\beta}=$ Estimated Coefficient Correlation; $\mathbf{S E}=$ Standard Error; Physical $\mathbf{Q o L}=$ Physical Quality of Life; Mental QoL = Mental Quality of Life; CES-D = Transformed Center for Epidemiologic Studies Depression Scale;Zidovudine (AZT); Nevirapine (NVP); lamivudine (3TC); Efavirenz (EFV). All the models were further adjusted for covariates listed in table 2.

$$
\begin{aligned}
& { }^{* *} \boldsymbol{P}<0.01 \\
& { }^{*} \boldsymbol{p}<0.05 \\
& { }_{\boldsymbol{p}}^{+}<0.1
\end{aligned}
$$

